\title{
Migraine and Bipolar Disorder
}

Ottavio Di Marco ${ }^{1 *}$, Carmine Di Marco ${ }^{2}$ and Stefania Di Mauro ${ }^{3}$

${ }^{1}$ Department of Psychiatry DSMPD, Ambulatorio Cefalee ASL Frosinone, Italy

${ }^{2}$ Student in Medicine, Università di Roma Tor Vergata, Italy

${ }^{3}$ Department of Psychiatry DSMP, ASL Frosinone, Italy

\begin{abstract}
The co-morbidity between migraine and bipolar disorders is well documented in numerous clinical and epidemiological studies, but the aetiologies are currently unknown. Many Authors have suggested some pathophysiological mechanisms in common. This co-morbidity, moreover, influences the outcome of both disorders. The aim of our work is to describe two case report, whose clinical history appears suggestive of the population subgroup with BD in comorbidity with migraine supposed in literature.
\end{abstract}

Keywords: Migraine; Bipolar disorder; Pathophysiological mechanisms

\section{Introduction}

Migraine is a complex, disabling disorder of the brain, characterised by recurrent headache, nausea, emesis, phonophobia and photophobia [1]. The prevalence of migraine is approximately $18 \%$ in women, $6 \%$ in men, and $4 \%$ in children [2] and its estimated heritability is between $40 \%$ and $65 \%[3,4]$. Migraine prevalence varies by age, sex, ethnic origin, and income.

Bipolar affective Disorder (BD) is a severe psychiatric illness affecting about $1-2.1 \%$ [5] of the population with significant morbidity and mortality. It is characterized by excessive shifts of mood, including periods of depression and mania.

The co-morbidity between migraine and $\mathrm{BD}$ is well documented in numerous clinical and epidemiological studies and there are clearly pathophysiological similarities. The lifetime prevalence of comorbid migraine among BD patients has been reported as $34.7 \%$ for females and $14.9 \%$ for males [6].

There seems to be a bidirectional relationship between migraine and BD. Patients suffering from migraine with aura are three times more likely to suffer from BD than the general population. Conversely, about one-third of patients with $\mathrm{BD}$ have migraine $[7,8]$. It has also been noted that migraine attacks are more frequent in sufferers of BPD compared to those of unipolar depression [9].

A retrospective analysis has identified that the presence of migraine in the course of $\mathrm{BD}$ is associated with earlier age at onset of $\mathrm{BD}$ and an unstable rapid cycling course [10], increased number of depressive episodes, and more severe depression [11]. Furthermore, a populationbased study $(n=36,984)$ has reported increased lifetime suicidal ideation and anxiety in $\mathrm{BD}$ subjects with comorbid migraine compared with those without the association [12]. These clinical differences support the proposal that the presence of comorbid migraine represents a more homogenous subtype of BD. [10]

The aetiology of both conditions are currently unknown. Many Authors have suggested common pathophysiology. Both migraine and $\mathrm{BD}$ have been linked to disturbances in the serortonergic [13], dopaminergic and glutaminergic systems [14], including voltage gated calcium channels. Evidence supports an important role of inflammation, oxidative and nitrosative stress in the pathophysiology of $\mathrm{BD}$ and migraine, with overlapping biological correlates of both disorders [15]. Abnormal activation cytokine-based inflammatory mechanisms has recently been reported in $\mathrm{BD}$ and in migraine headache [16]. Other Authors supposed that genetic factors influence susceptibility to both illnesses [10].

A family history of mood disorder and migraine in first-degree relatives was also reported to be associated with an increased risk of comorbidity [17]. In addition, the effectiveness of anti-epileptic treatments for both $\mathrm{BD}$ and migraine headache provides an indication that there is a link in pathophysiological processes between the two disorders [18]. These strong links between migraine and bipolar disorder, suggest either common etiology or co-morbid migraine as a sub-phenotype of bipolar disorder.

The aim of our work is to describe two case report, whose clinical history appears suggestive of the population subgroup with $\mathrm{BD}$ in comorbidity with migraine supposed in literature.

\section{Case Report}

The first case report concerns a woman of 53 years old. Her onset of migraine was at 15 years old, with $6 / 7$ attacks on month, but she didn't consult any specialist. At the same period she started to use substance, as cannabis. Three years later, at 18 years old, the depressive symptomatology has arisen with an attempted suicide in the first month after the onset of psychiatric disorder. Because of the severe depression and of attempted suicide she was hospitalized in Psychiatric Unit and she was treated with Benzodiazepines and Antidepressants Drugs. The patient describes an improvement in mood. She started University and after three years she suspended the therapy. At 22 years old she married and after one year the son was born. At 25 years she returned to study at University. In the following period the patient presented a high level of stress due to university exams, with a new worsening of migraine and anxious symptomatology and she make occasional resort to Benzodiazepine. She consulted Specialist in Psychotherapy and Neurology. The Neurologist prescribed to the patient some drugs, as

*Corresponding author: Dr. Ottavio Di Marco, Department of Psychiatry DSMPD, Ambulatorio Cefalee ASL Frosinone, Italy; Tel: +393383695817; E-mail: ottavio.dimarco@virgilio.it

Received December 05, 2018; Accepted December 24, 2018; Published December 30, 2018

Citation: Marco OD, Marco CD, Mauro SD (2018) Migraine and Bipolar Disorder. J Neurol Neurophysiol 9: 477. doi:10.4172/2155-9562.1000477

Copyright: (c) 2018 Marco OD, et al. This is an open-access article distributed under the terms of the Creative Commons Attribution License, which permits unrestricted use, distribution, and reproduction in any medium, provided the original author and source are credited. 
Valproate, Antidepressant, Topiramate, but she presented numerous side effects responsible for continues changes in therapy. After some years, the Neurologist dead and she stopped the therapy, but changed Psychotherapist. At 35 years old she started to work in another city and at the same time she presented relationship problems with her husband and in family management. Symptoms such as anxiety, emotional lability and worsening of migraine appeared again, with a frequency of 15-18 days for month. She took anti-inflammatores and triptans and started a new psychotherapeutic treatment, but with little benefit. At 42 years old she was diagnosed with autoimmune disease and the migraine worsened again. The mood became increasingly irritable and dysphoric, marked insomnia and increased activity appeared. She started to be absent from work, until an aggression episode that required a second hospitalization in Psychiatric Unit for 15 days. At discharge, she took therapy with Valproate and Quetiapina, but migraine was present with the same frequency (15-18 days for month) and mood remained unstable. She increased weight and therefore suspended therapy alone. She rejected other mood stabilizers. At 48 years old she presented another maniac episode and frequency migraine was always 15-18 days for month. She consulted another Neurologist. After the clinical-anamnestic evaluations she was discharged with the diagnosis of Bipolar Disorder in comorbidity with chronic migraine. The Specialist prescribed her Carbolithium for mood disorder and botulinum treatment for migraine. At the follow-up visit she reported improvement of both disorders.

The second case report is about a woman of 47 years. She reports the onset of cephalalgia symptomatology at about 20 years, with a headache frequency of 4-5 days per month. Because of the same, he made several visits to the Emergency Unit, with diagnosis of anxiety disorder with somatization and treated with occasional use of Benzodiazepines. The patient describes herself as tendentially anxious since adolescence. At the age of 28 years old, she presented a severe depressive episode, for which of an entity that required two hospitalizations in the psychiatric field.

At discharge, she was prescribed antidepressant therapy, with gradual improvement in mood, but with headache stability in terms of frequency (always 4-5 days/month). This therapy was taken for about 2 years, with decrease until suspension because the patient wanted a pregnancy. At 34, the daughter was born, in abs. Remember the period after the birth of the daughter as very stressful, with worsening of insomnia, even for the needs of caring for the child, so continued to make occasional resort to Benzodiazepine and hypnoinductive.

After about 10 months from the birth he presented an episode characterized by restlessness, easy irritability and a tendentially dysphoric mood, increased activity aimed, insomnia not responding to benzodiazepines, acceleration of the ideic flow, which however presented themes of greatness. Because of the symptoms described above, she was re-admitted to the competent Psychiatric Service and discharged after about 20 days with lithium salts therapy, benzodiazepines and atypical antipsychotics and with a diagnosis of mood bipolar disorder, the most recent manic episode. According to the diagnostic criteria of the DSM, after returning to his home, after about two months from the hospitalization, he appreciated a tone of humor substantially in line, not perceptive changes or the content of the thought, with a good efficiency in the performance of daily tasks. Insomnia persisted, characterized by frequent nocturnal awakenings described by the patient associated with headache.

The specialist in psychiatry that followed her, secondarily to the reported insomnia, has increased her Benzodiazepines and antipsychotics, considering insomnia and headache symptoms of somatization secondary to Mood Disorder. in the next months she has consulted other Specialists in Psychiatry for the persistence of both insomnia and headache. The frequency of the cephalalgia crises, which became multi-weekly, and the intensity of the same had progressively increased. This was associated with a fair amount of irritability due to sleep deprivation, with various changes in dosage and type of psychiatric drugs. At 41, she was re-admitted to the SPDC due to irritability, which however did not meet the criteria for a new episode of Mood Disorder. After the clinical-anamnestic evaluations she was discharged with the diagnosis of Bipolar Disorder in comorbidity with chronic migraine and treated with Topiramato. At the follow-up visit, an overall improvement in the psychopathological picture was appreciated, with a reduction in the intensity and frequency of migraine attacks (3 days per month), as well as a global improvement in the quality of life [19].

\section{Discussion}

In our study we report two case reports, both about of women. As typically occurs, the onset of migraine precedes that of psychiatric disorder [20]. In both cases, the psychiatric symptomatology at onset was a depressive episode, in the first case report at 18 years old and in the second at 28 years old. These data are in agreement with those present in the literature. Many Authors, indeed, reported that the onset of psychiatric disorder is preferentially associated with an index episode of depression rather than mania [16]. Moreover, some studies have reported an increased lifetime suicidal ideation and anxiety in $\mathrm{BD}$ subjects with comorbid migraine compared with those without the association [12]. In the first case, there was a suicidal attempt at the onset of depression at 18 years old. The severity of symptomatology and this attempt caused an admission in Psychiatric Unit.

Both patients receive at the start antidepressant drugs and Benzodiazepine. In both patients the association of migraine and Bipolar Disorder comorbidity has involved worse outcomes. Both patients, indeed, had high frequency of migraine episodes and this frequency worsened in the presence of psychiatric symptoms. On the other hand, both patients were getting worse in working and social functioning. Only in a second time the patients received therapy with mood stabilizers including valproate or lithium, secondly the occurrence of irritability and insomnia, with an improvement of psychiatric and migraine symptomatology. This improvement was observed also in the follow-up visits.

In according with literature, migraine in depressed patients could represent a trait in the bipolar spectrum and the timeliness of the treatment could improve the outcome. All these data suggest the opportunity that the Clinician, in front of a patient with severe depressive symptomatology at the onset, investigates in depth the presence of migraine comorbidity. This observation is important in the first for a correct diagnosis, because it could be the onset of a BD and not of a Unipolar Disorder. Second, for a timely treatment with mood stabilizers. This treatment, according with literature, improves the outcome of both diseases.

\section{Conclusion}

It is very important to recognize and diagnose the comorbidity between $\mathrm{BD}$ and migraine, because these patients may represent a subgroup of $\mathrm{BD}$, with a worse prognosis. A thorough medical history is therefore necessary. We wont to underline the need of more studies about of this comorbidity, also to set up a suitable pharmacological treatment and this may ultimately also influence the therapeutic outcome towards prescribed medications. 


\section{References}

1. Davidoff RA (2002) Migraine: Manifestations, pathogenesis and management. Oxford University Press, New York.

2. Silberstein SD, Goadsby PJ (2002) Migraine: Preventive treatment. Cephalalgia 22: 491-512.

3. Holland J, Agius M (2011) Neurobiology of bipolar disorder-lessons from migraine disorders. Psychiatr Danub 23: 162-165.

4. Schürks M, Rist PM, Kurth T (2010) 5-HTTLPR polymorphism in the serotonin transporter gene and migraine: A systematic review and meta-analysis. Cephalalgia 30: 1296-1305

5. Merikangas KR, Akiskal HS, Angst J (2007) Lifetime and 12- month prevalence of bipolar spectrum disorder in the national comorbidity survey replication. Arch Gen Psychiatry 64: 543-552.

6. Mclntyre RS, Konarski JZ, Wilkins K, Bouffard B, Soczynska JK, et al. (2006) The prevalence and impact of migraine headache in bipolar disorder: Results from the Canadian community health survey. Headache 46: 973-982.

7. Merikangas KR, Merikangas JR, Angst J (1993) Headache syndromes and psychiatric disorders: Association and familial transmission. J Psychiatr Res 27: 197-210.

8. Breslau N, Davis GC, Andreski P (1991) Migraine, psychiatric disorders, and suicide attempts: An epidemiologic study of young adults. Psychiatry Res 37 : $11-23$

9. Fasmer OB (2001) The prevalence of migraine in patients with bipolar and unipolar affective disorders. Cephalalgia 21: 894-899.

10. Gordon-Smith K, Forty L, Chan C (2015) Rapid cycling as a feature of bipolar disorder and comorbid migraine. J Affect Disord 175: 320-324.
11. Saunders EFH, Nazir R, Kamali M (2014) Gender differences, clinical correlates and longitudinal outcome of bipolar disorder with comorbid migraine. J Clin Psychiatry 75: 512-519.

12. Nguyen TV, Low NC (2013) Comorbidity of migraine and mood episodes in a nationally representative population-based sample. Headache 53: 498-506.

13. Hamel E (2007) Serotonin and migraine: Biology and clinical implications. Cephalalgia 27: 1295-1300.

14. Vaccaro M, Riva C, Tremolizzo L, Longoni M, Aliprandi A, et al. (2007) Platelet glutamate uptake and release in migraine with and without aura. Cephalalgia 27: $35-40$.

15. da Costa SC, Passos IC, Réus IG, Carvalho AF, Soares JC, et al. (2016) The comorbidity of bipolar disorder and migraine: The role of inflammation and oxidative and nitrosative stress. Current Molecular Medicine 16: 179186.

16. Brietzke E, Mansur RB, Grassi-Oliveira R, Soczynska JK, McIntyre RS, et al (2012) Inflammatory cytokines as an underlying mechanism of the comorbidity between bipolar disorder and migraine. Med Hypotheses 78: 601-605

17. Franchini L, Bongiorno F, Dotoli D, Rainero I, Pinessi L, et al. (2004) Migraine headache and mood disorders: a descriptive study in an out- patient psychiatric population. J Affect Disord 81: 157-160.

18. Engmann B (2012) Bipolar affective disorder and migraine. Case Rep Med 2012: 389-851.

19. di Marco O, di Mauro S (2018) Bipolar disorder and chronic migraine: An open problem. Neurological Sciences 39: 85-86.

20. Ortiz A, Cervantes P, Zlotnik G, vandeVelde C, Slaney C, et al. (2010) Crossprevalence of migraine and bipolar disorder. Bipolar Disord 12: 397-403. 